\section{Cureus}

Received 04/02/2017

Review began 04/19/2017

Review ended 05/01/2017

Published 05/04/2017

๑) Copyright 2017

Abbass et al. This is an open access article distributed under the terms of the Creative Commons Attribution License CC-BY 3.0., which permits unrestricted use, distribution, and reproduction in any medium, provided the original author and source are credited.

\title{
Liddle Syndrome in Association with Aortic Dissection
}

\author{
Aamer Abbass ${ }^{1}$, Jason D'Souza ${ }^{2}$, Sameen Khalid ${ }^{1}$, FNU Asad-Ur-Rahman ${ }^{3}$, Joseph Limback ${ }^{4}$ \\ , Jeremy R. Burt ${ }^{5}$, Rajesh Shah ${ }^{6}$ \\ 1. Internal Medicine Residency, Florida Hospital-Orlando, Orlando, USA 2. Internal Medicine, Florida \\ Hospital-Orlando 3. Gastroenterology, Cleveland Clinic Florida, Weston, Select Country 4. Diagnostic \\ Radiology, Florida Hospital-Orlando 5. Diagnostic Radiology, Florida Hospital-Orlando, Orlando, USA 6. \\ Orlando Cardiac \& Vascular Specialists, Florida Hospital-Orlando
}

$\square$ Corresponding author: Aamer Abbass, aamikem@gmail.com

Disclosures can be found in Additional Information at the end of the article

\section{Abstract}

Liddle syndrome is a rare form of autosomal dominant monogenic hypertension manifested as an early onset of resistant hypertension with either no response or suboptimal response to conventional antihypertensive therapy. If there is a delay in diagnosis, uncontrolled hypertension can lead to end organ damage. To our knowledge, aortic dissection has not been reported in association with this disease. We report a case of a dissecting aortic aneurysm occurring in association with Liddle syndrome.

Categories: Cardiac/Thoracic/Vascular Surgery, Cardiology, Nephrology

Keywords: aortic dissection, liddle syndrome, premature hypertension

\section{Introduction}

Liddle syndrome (LS) is a rare form of monogenic hypertension first described by Sir Grant Liddle in 1963 [1]. It is perceived to be a very rare disease with a reported prevalence of < $1 / 1,000,000$ at present [2]. Up until 2008, only 30 patients affected with this monogenetic disorder had been reported in the world [9]. Two small single-center studies have estimated the prevalence to be about $1.52 \%$ and $6 \%$ among hypertensive patients with genetic testing and phenotypical LS, respectively [3-4]. Our knowledge about LS is evolving, given its relative rarity. It typically manifests as early onset of resistant hypertension with either no response or suboptimal response to conventional antihypertensive therapy. Therefore, uncontrolled hypertension can lead to target organ dysfunction, such as stroke, heart failure, retinopathy, end-stage renal disease, etc. To our knowledge, aortic dissection has not been reported in association with this disease. We report a case of dissecting aortic aneurysm occurring in association with Liddle syndrome.

\section{Case Presentation}

A 29-year-old Caucasian male with an established history of Liddle syndrome, diagnosed at the age of 8 , and a 10-pack-year smoking history, presented to a local hospital with the sudden onset mid-sternal chest pain radiating to the back. The chest pain was associated with lightheadedness, headache, diaphoresis, palpitations, nausea, and one episode of non-bloody emesis. Family history was significant for LS in his father, younger brother, and paternal uncle. His father and paternal uncle met an early demise secondary to intracerebral bleeds from elevated blood pressure related to LS. In addition, he also confessed to being non-compliant with his antihypertensive medications for many years. His initial vitals were a temperature of 


\section{Cureus}

98.2 F, pulse rate of 83 beats/min, respiratory rate of 19 breaths/min, oxygen saturation of $98 \%$ on room air, and a blood pressure (BP) of 220/120 mmHg. His BP was equal in bilateral upper extremities with strong bilateral pedal pulses. A 12-lead electrocardiogram performed in emergency room revealed evidence of a left ventricular hypertrophy without any acute ST-T wave changes (Figure 1).

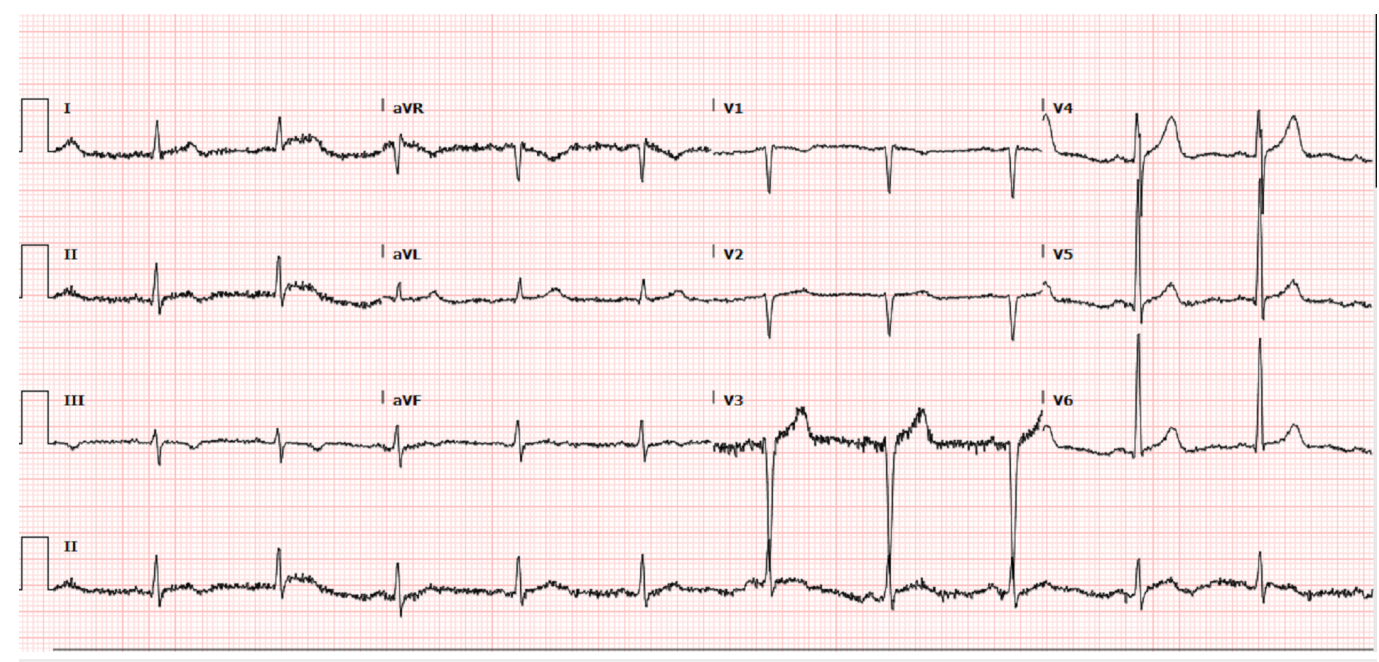

\section{FIGURE 1: 12-lead electrocardiogram with evidence of left ventricular hypertrophy}

Given his concerning constellation of symptoms and findings on physical examination, a stat computerized tomographic angiogram (CTA) of the chest was performed, which showed an acute penetrating ulcer in the proximal descending thoracic aorta with intramural hematoma extending from the origin of the left subclavian artery into the suprarenal abdominal aorta, consistent with Stanford Type B aortic dissection (Figures 2-3). The intramural hematoma extended to the origin of the celiac artery resulting in a high-grade stenosis (Figure 4). CTA of the abdomen and pelvis also revealed fusiform dilatation of the suprarenal abdominal aorta with a maximal diameter of $3.4 \times 3.3 \mathrm{~cm}$.

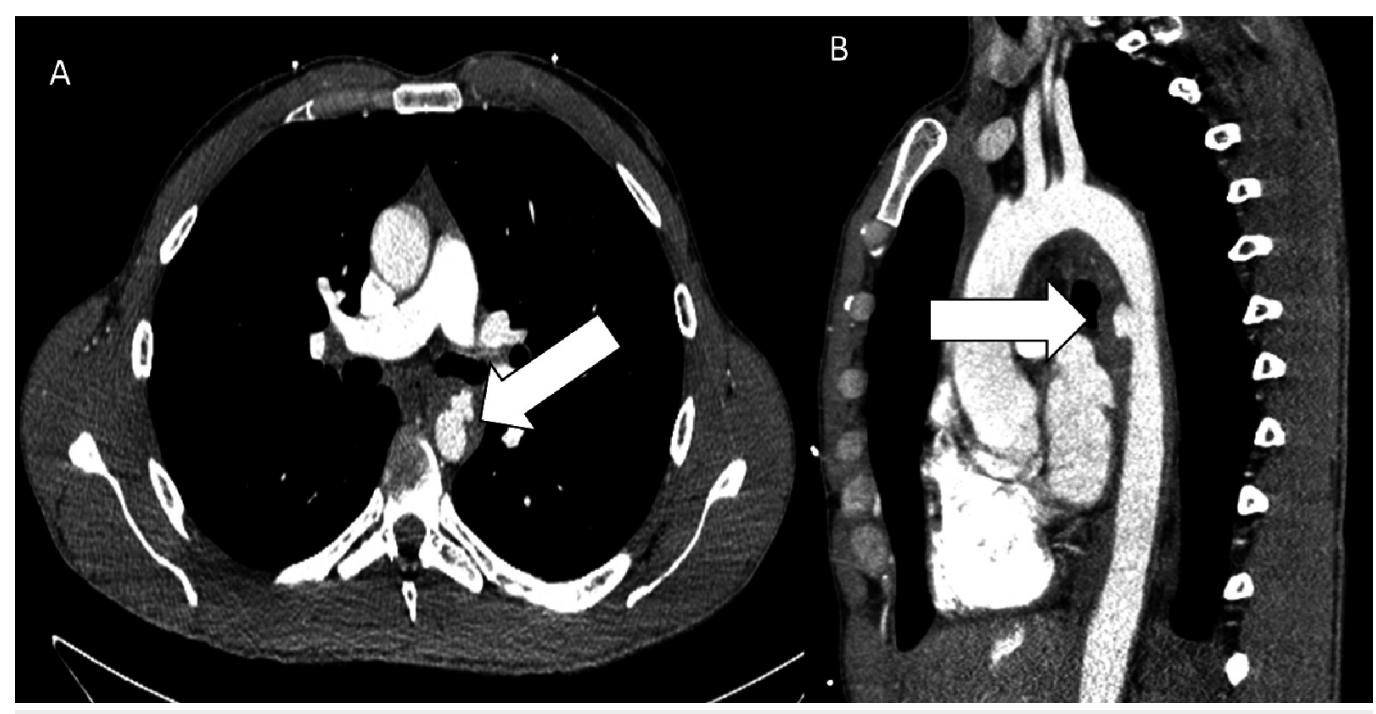

FIGURE 2: Computed tomography (CT) of the mid-chest

A) Axial contrast-enhanced CT of the mid-chest showing dilation of the descending aorta with an 


\section{Cureus}

intramural hematoma and penetrating ulcer (arrow) in the proximal descending aorta. B) Sagittal oblique CT showing the intramural hematoma and penetrating ulcer (arrow).

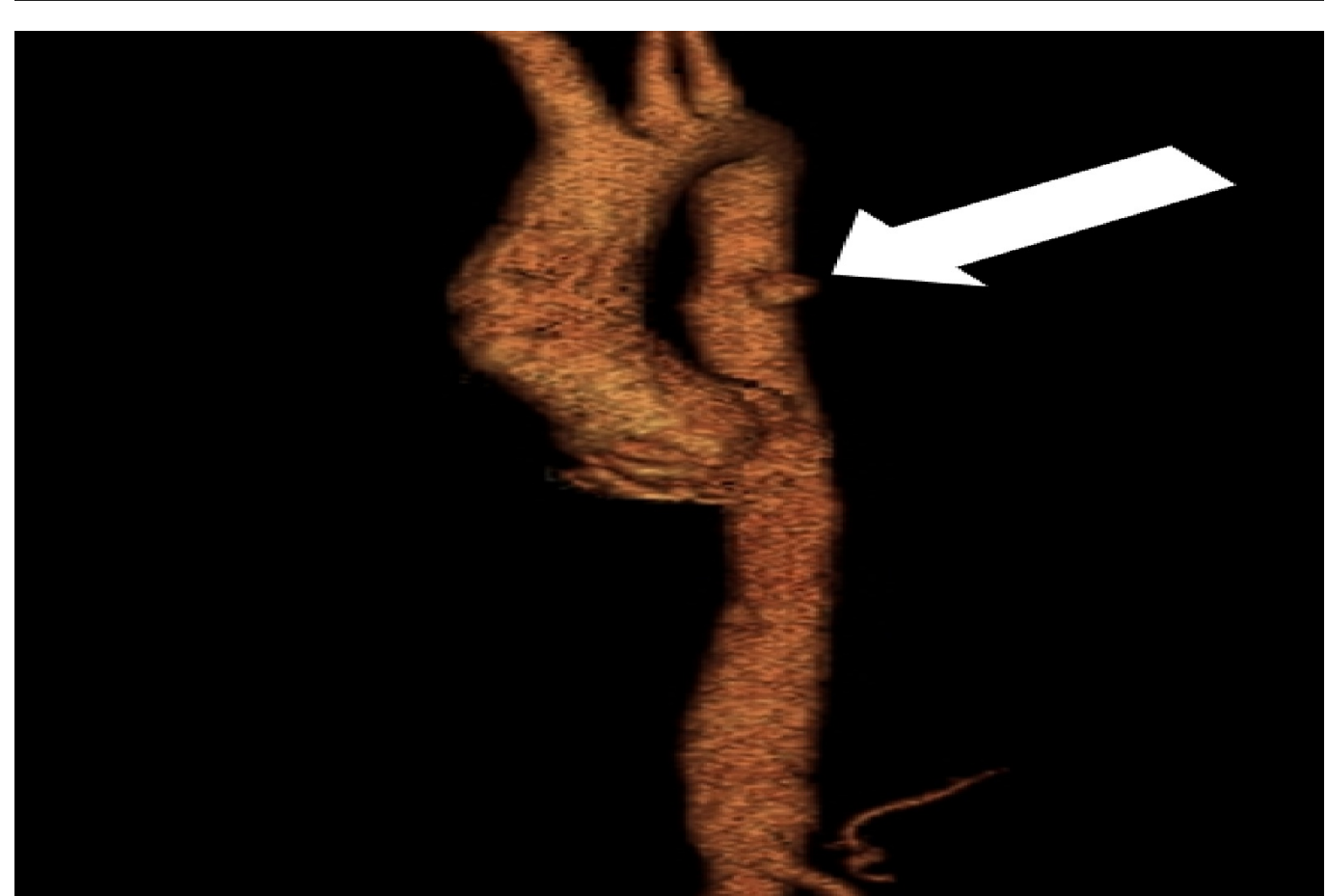

FIGURE 3: Volume-rendered computed tomography angiogram (CTA) demonstrating the penetrating ulcer (arrow).

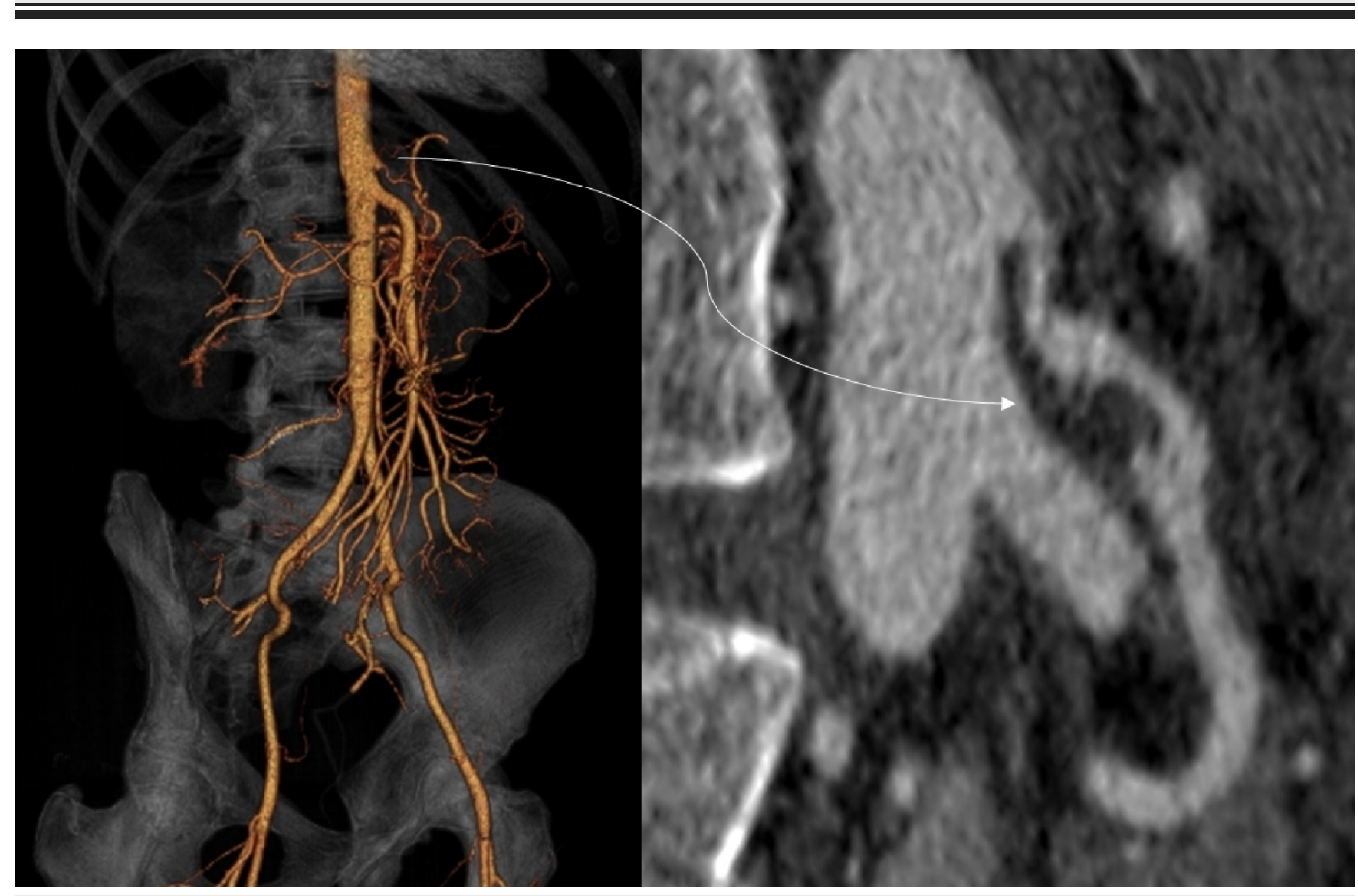

FIGURE 4: Volume-rendered and curviplanar reformatted computed tomography

Images showing extension of the intramural hematoma into the celiac artery. This caused mild- 


\section{Cureus}

moderate stenosis.

The patient was immediately started on intravenous labetalol and nitroprusside drip for heart rate and blood pressure control and was subsequently transferred to our hospital for further management and hemodynamic monitoring. His initial lab work was notable for a sodium of $134 \mathrm{mEq} / \mathrm{L}$, potassium of $3.9 \mathrm{mEq} / \mathrm{L}$, chloride of $99 \mathrm{mEq} / \mathrm{L}$, bicarbonate of $19 \mathrm{mEq} / \mathrm{L}$, blood urea nitrogen (BUN) of $16 \mathrm{mg} / \mathrm{dl}$, and creatinine of $0.84 \mathrm{mg} / \mathrm{dl}$. Routine echocardiography demonstrated a normal ejection fraction of 60-65\% with left ventricular hypertrophy. The patient required continuous intravenous infusions of various BP medications, including labetalol, nitroprusside, and nitroglycerin to achieve target BP goals in first 24-48 hours. However, it was not until the patient was started on $20 \mathrm{mg}$ of oral amiloride that he was weaned off some of the anti-hypertensive drips. In addition, the patient also required clonidine, metoprolol, nifedipine, hydralazine, and isosorbide mononitrate to strictly maintain his BP at goal. By Day 3 of his hospitalization, BP control was achieved with an oral antihypertensive regimen as mentioned above and all the intravenous drips were discontinued. The patient remained asymptomatic during the ICU stay.

A cardiothoracic consultation was obtained and medical management was the initial recommendation. However, on hospital Day 6, a repeat CTA of the chest, abdomen, and pelvis was performed to confirm stabilization of the penetrating ulcer. It revealed that the intramural hematoma in the mid-thoracic aorta had formed a deep penetrating ulcer, in addition to stable fusiform dilatation of the suprarenal abdominal aorta with a maximal diameter of $3.4 \times 3.3 \mathrm{~cm}$ (Figure 5).

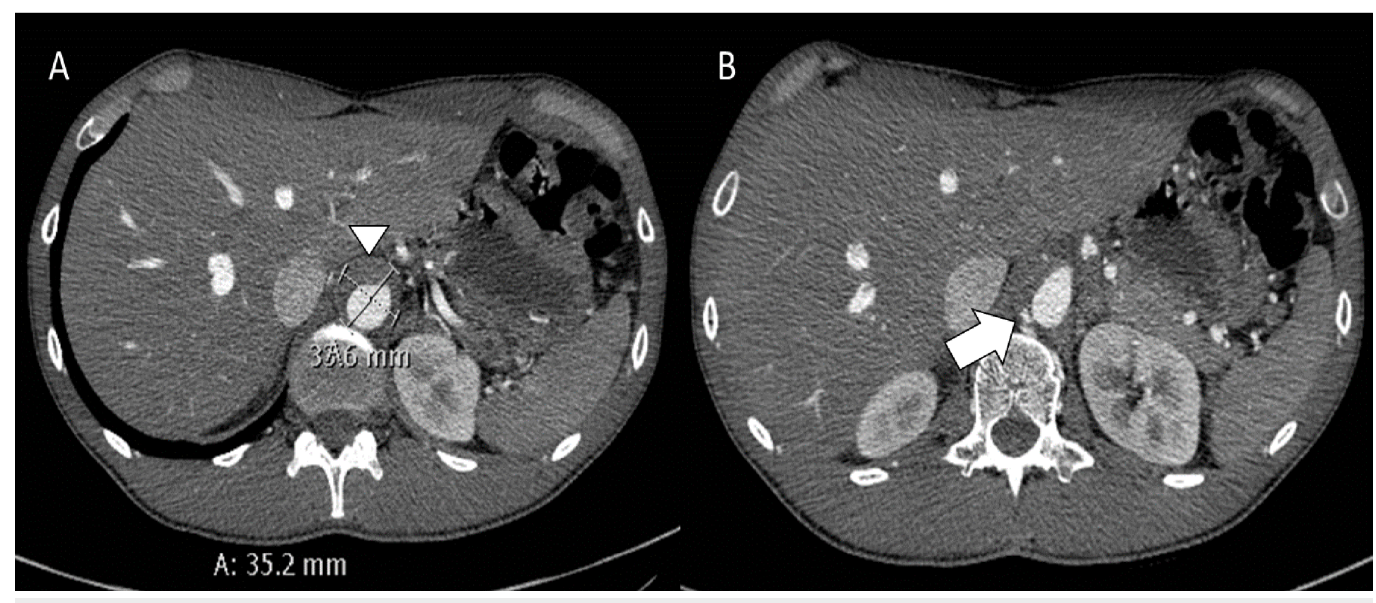

FIGURE 5: Axial contrast-enhanced computed tomography (CT) of the upper abdomen

A) CT images showing an intramural hematoma with an aneurysm (arrowhead). B) Axial CT showing another small penetrating ulcer (white arrow).

Vascular surgery was consulted and the patient underwent thoracic endovascular aortic repair (TEVAR) with the exclusion of the intramural hematoma and penetrating ulcer in the middescending thoracic aorta using a Gore ${ }^{\circledR}$ TAG ${ }^{\circledR}$ Thoracic Endoprosthesis 26 x 10 (WL Gore \& Associates, Inc., Flagstaff, AZ) on hospital Day 8 . The patient had an uneventful recovery and was discharged on multiple oral BP medications as outlined above after 10 days of hospitalization. The patient followed up in the clinic one month after discharge from the 
hospital and reported feeling well with no further episodes of chest pain. His blood pressure had been well controlled on multiple oral antihypertensive medications, including amiloride.

\section{Discussion}

Liddle syndrome is a rare form of hereditary monogenic hypertension. It is caused by a mutation of either $\beta$ or $\gamma$ subunits of SCCN1B and SCCN1G genes, which prevents degradation of the epithelial sodium channel (ENaC) of distal convoluted tubule from proteasomes, resulting in constitutive activation of the ENaC. This constitutive activation causes unregulated sodium and water reabsorption and volume expansion leading to uncontrolled hypertension [5]. The result of this cascade of events is a negative feedback of the hormonal axis leading to a low renin-low aldosterone state, and hence, the term pseudo-aldosteronism. Hypokalemia and metabolic alkalosis associated with this feedback mechanism is the norm rather than the rule and provides important diagnostic clues [6-7]. Therefore, normal serum chemistries, such as in our patient, can be expected.

Target organ dysfunction is not an uncommon complication, given the early age of onset and longstanding uncontrolled hypertension, as the condition is not readily diagnosed and treatment differs from essential hypertension. Under-recognition and inappropriate treatment can lead to sustained hypertension and associated complications, including (but not limited to) renal failure, hypertensive heart disease, cerebrovascular mortality, retinal damage, and, in severe cases, premature and sudden death prior to the age of 40 [8]. To the best of our knowledge, our patient is the index case of Liddle syndrome presenting with aortic dissection. Diagnostic clues include a positive family history, characteristic lab findings, the lack of response to conventional antihypertensives but a dramatic response to ENaC blockers, and genetic testing consistent with the underlying mutation. Differential diagnosis includes glucocorticoid-remediable aldosteronism, apparent mineralocorticoid excess, primary aldosteronism, Cushing's syndrome, pheochromocytoma, renovascular hypertension, essential hypertension with diuretic use, and congenital adrenal hyperplasia. Treatment options are limited. However, most patients demonstrate a dramatic and remarkable response to a saltrestricted diet and ENaC blockers, such as amiloride and triamterene. Amiloride competes with sodium at the level of the ENaC conducting pore to prevent sodium reabsorption [9].

\section{Conclusions}

Although readily treatable, Liddle syndrome is frequently underdiagnosed and widely misrecognized. Genetic testing is recommended for patients and first-degree relatives to uncover existing and de-novo mutations. Although LS is considered to be a rare condition, increased awareness and more frequent genetic testing may lead to increased reported prevalence.

\section{Additional Information Disclosures}

Human subjects: Consent was obtained by all participants in this study. Conflicts of interest: In compliance with the ICMJE uniform disclosure form, all authors declare the following: Payment/services info: All authors have declared that no financial support was received from any organization for the submitted work. Financial relationships: All authors have declared that they have no financial relationships at present or within the previous three years with any organizations that might have an interest in the submitted work. Other relationships: All authors have declared that there are no other relationships or activities that could appear to have influenced the submitted work. 


\section{References}

1. Liddle GW, Bledsoe T, Coppage WS Jr: A familial renal disorder simulating primary aldosteronism but with negligible aldosterone secretion. Trans Assoc Am Physicians. 1963, 76:199-213.

2. The portal for rare diseases and orphan drugs . (2017). Accessed: $2 / 3 / 2017$ : http://www.orpha.net/consor/cgi-bin/Disease_Search.php? lng=EN\&data_id=927 \&Disease_Disease_Search_diseaseGroup=liddle-...

3. Wang LP, Yang KQ, Jiang XJ, et al.: Prevalence of Liddle syndrome among young hypertension patients of undetermined cause in a Chinese population. J Clin Hypertens (Greenwich). 2015, 17:902-7. 10.1111/jch.12598

4. Tapolyai M, Uysal A, Dossabhoy NR, et al.: High prevalence of Liddle syndrome phenotype among hypertensive US veterans in Northwest Louisiana. J Clin Hypertens (Greenwich). 2010, 12:856-60. 10.1111/j.1751-7176.2010.00359.x

5. Yang KQ, Xiao Y, Tian T, et al.: Molecular genetics of Liddle's syndrome . Clin Chim Acta. 2014, 436:202-6. 10.1016/j.cca.2014.05.015

6. Warnock DG: Liddle syndrome: genetics and mechanisms of $\mathrm{Na}+$ channel defects . Am J Med Sci. 2001, 322:302-7. 10.1097/00000441-200112000-00002

7. Gao L, Wang L, Liu Y, et al.: A family with Liddle syndrome caused by a novel missense mutation in the PY motif of the beta-subunit of the epithelial sodium channel. J Pediatr. 2013, 162:166-70. 10.1016/j.jpeds.2012.06.017

8. Kota SK, Kota SK, Panda S, et al.: A case of Liddle's syndrome; unusual presentation with hypertensive encephalopathy. Saudi J Kidney Dis Transpl. 2014, 25:869-71. 10.4103/13192442.135185

9. Rossier BC, Schild L: Epithelial sodium channel: mendelian versus essential hypertension . Hypertension. 2008, 52:595-600. 10.1161/HYPERTENSIONAHA.107.097147 